\title{
CINÉTICA DE CRECIMIENTO DE Lactobacillus lactis Y DETERMINACIÓN DEL EFECTO PROBIÓTICO EN CEPAS PATÓGENAS ${ }^{1}$
}

Henry Jurado-Gámez²

Verónica Jarrín-Jarrín ${ }^{3}$

\section{RESUMEN}

Introducción: Las bacterias ácido-lácticas se han estudiado por su capacidad de crecer en ambientes difíciles y generar antagonismos con otros microorganismos. La presente investigación buscó determinar los parámetros de la cinética de crecimiento de Lactobacillus lactis y su efecto probiótico sobre Escherichia coli, Salmonella typhimurium, Clostridium perfringens y Staphylococcus aureus. Materiales y Métodos: Se evaluó el crecimiento de la cepa láctica a concentraciones de $0,5,1$ y $2 \%$ de sales biliares; 1 y $1,5 \%$ de bilis; tres niveles de $\mathrm{pH}(2,5,4,5$ y 7$)$ y dos de temperaturas $\left(38\right.$ y $\left.45^{\circ} \mathrm{C}\right)$. También se evalúo la susceptibilidad de todas las cepas a los antibióticos Dicloxacilina, Cefepime, Cefalotina, Ciprofloxacina, Pentamicina y Penicilina con la técnica de Kirby-Bauer. Se determinó la inhibición de L. lactis y su sobrenadante en $E$. coli, S. typhimurium, C. perfringens y S. aureus y se estimaron los parámetros de: crecimiento, $\mathrm{pH}$, consumo de azúcar, acidez y consumo de proteína durante la cinética de fermentación en medio MRS. Se determinaron péptidos presentes en el sobrenadante de L. lactis mediante HPLC. Resultados: Se obtuvo un crecimiento de $1,2 \mathrm{x}$ $10^{9}$ y $4,1 \times 10^{9} \mathrm{ufc} / \mathrm{ml}$ a $1,2 \%$ de bilis y a $0,5 \%$ de sales biliares; $1,1 \times 10^{11}, 2,1 \times 10^{10}$ y $1,0 \times 10^{10}$ ufc/ml para $\mathrm{pH} 2,5,4,5$ y 7 respectivamente; $\mathrm{y}$ $1,7 \times 10^{13}$ y $1,4 \times 10^{13} \mathrm{ufc} / \mathrm{ml}$ para 45 y $38^{\circ} \mathrm{C}$. La cepa láctica presentó resistencia a Dicloxacilina y Cefalotina; C. perfringens, a Dicloxacilina y Penicilina; S. typhimurium y E. coli, a Cefalotina; y $S$. aureus, a Dicloxacilina. L. lactis inhibió a $E$. coli y C. perfringens, y el sobrenadante incluyó a $S$. aureus. Conclusión: Se concluye que L. lactis presenta adecuados crecimientos en condiciones in vitro e inhibió a E. coli, C. perfringens y S. aureus pero no a $S$. typhimurium.

Palabras clave: probiótico, crecimiento, antagonismo microbiano, bacteria patógena, Lactobacillus lactis.

\section{Lactobacillus lactis GROWTH KINETICS AND DETERMINATION OF PROBIOTIC EFFECT ON PATHOGEN STRAINS}

\begin{abstract}
Introduction: Acid lactic bacteria have been studied for their ability to grow in harsh environments and generate antagonisms with other microorganisms. The present study
\end{abstract}

\footnotetext{
${ }^{1}$ Proyecto financiado por la Vicerrectoría de Postgrados y Relaciones Internacionales (VIPRI) de la Universidad de Nariño. 2 Ph.D. en Ingeniería de Alimentos. Universidad de Nariño, Facultad de Ciencias Pecuarias, Departamento de Producción y Procesamiento Animal, Programa de Zootecnia - Grupo de Investigación Fise-Probiotec. Pasto, Colombia. Correo electrónico: henryjugam@gmail.com

3 M.Sc. Universidad de Nariño, Facultad de Agroindustria, Programa de Agroindustria. Pasto, Colombia. Correo electrónico: Jarrin.veronica@gmail.com
} 
sought to determine the kinetic parameters of growth of Lactobacillus lactis and its effect on Escherichia coli, Salmonella typhimurium, Clostridium perfringens and Staphylococcus aureus. Materials and Methods: The growth of the lactic strain was evaluated at concentrations of $0.5,1$ and $2 \%$ bile salts; 1 and $1.5 \%$ bile; three levels of $\mathrm{pH}(2.5,4.5$ and 7$)$ and two temperatures $\left(38\right.$ and $\left.45^{\circ} \mathrm{C}\right)$. Susceptibility of all strains to Dicloxacillin, Cefepime, Cephalothin, Ciprofloxacin, Gentamicin and Penicillin was also evaluated using Kirby-Bauer technique. Inhibition of L. lactis and its supernatant in E.coli, S. typhimurium, S. aureus and C. perfringens and the following parameters were determined and estimated: growth, $\mathrm{pH}$, sugar consumption, acidity and protein consumption during the kinetic fermentation in MRS. Peptides present in the L. lactis supernatant through HPLC were

\section{INTRODUCCIÓN}

Las bacterias ácido-lácticas son un grupo de bacterias Gram-positivas que tiene como característica común la producción de ácido láctico a través de la fermentación de carbohidratos (1). Además, son microorganismos catalasa negativos y no productores de gas (2). Dentro de este grupo, los más importantes pertenecen al género Lactobacillus, conocidos como GRAS (Generalmente Reconocidos como Seguros) y considerados benéficos por su capacidad de digerir proteína, carbohidratos y grasas de los alimentos, cualidad que les permite ayudar al organismo huésped en la absorción de nutrientes esenciales como minerales, aminoácidos y vitaminas (3). Otra característica importante es su capacidad para inhibir otros microorganismos como bacterias patógenas causantes de problemas a nivel agroindustrial y sanitario (4). determined. Results: Growth of $1.2 \times 10^{9}$ and $4.1 \times 10^{9} \mathrm{CFU} / \mathrm{ml}$ at $1.2 \%$ bile and $0.5 \%$ bile salt was obtained; $1.1 \times 10^{11}, 2.1 \times 10^{10}$ and $1.0 \times 10^{10}$ $\mathrm{CFU} / \mathrm{ml}$ for $2.5,4.5$ and $7 \mathrm{pH}$ respectively; and $1.7 \times 10^{13}$ and $1.4 \times 10^{13} \mathrm{CFU} / \mathrm{ml}$ for 45 and $38^{\circ} \mathrm{C}$. The lactic strain showed resistance to Cephalothin and Dicloxacilin; C. perfringens, to Penicillin and Dicloxacillin; S. typhimurium and E. coli to Cephalothin; and S. aureus, to Dicloxacillin. L. lactis inhibited E. coli and $C$. perfringens, and the supernatant included $S$. aureus. Conclusion: It is concluded that L. lactis present adequate growth in conditions in vitro and inhibited E. coli, C. perfringens and S. aureus but not $S$. typhimurium.

Key words: probiotic, growth, microbial antagonism, pathogenic bacteria, Lactobacillus lactis.

Los principales mecanismos antagónicos de las bacterias ácido-lácticas son su capacidad de reducir el $\mathrm{pH}$ del medio, la producción de biocinas y la producción de ácidos orgánicos (5). El primero genera un ambiente inadecuado para el crecimiento de otros microorganismos, que necesitan $\mathrm{pH}$ cercanos a la neutralidad para sobrevivir en el medio (6). Las biocinas o bacteriocinas son sustancias proteínicas que actúan sobre la pared celular de otras bacterias generando la muerte, estas se han hecho importantes debido a su aplicación en la preservación de alimentos con la consecuente reducción de preservantes químicos $(7,8)$. Los ácidos orgánicos aumentan la acidez del medio e inhiben el crecimiento de otras bacterias (9).

Las condiciones gastrointestinales de los animales domésticos son un reto para toda bacteria que necesite colonizarlo; los cambios en el $\mathrm{pH}$, los niveles de temperatura y la secreción 
de bilis y sales biliares son barreras para un crecimiento adecuado de los microorganismos en el sistema digestivo (10). La evaluación de cepas probióticas requiere demostrar su viabilidad a estas condiciones, ya que la vía principal de administración de estos microorganismos es oral (11). Las evaluaciones in vitro permiten tener un primer acercamiento al potencial de la cepa como agente probiótico que pueda dar paso a una investigación a nivel de campo.

Los antibióticos son utilizados en el tratamiento de enfermedades bacterianas, sin embargo, las bacterias son seres vivos con una alta adaptabilidad y pueden desarrollar resistencia a los antibióticos (12). La resistencia es un factor natural de las bacterias, pero este proceso se ha visto acelerado por el uso indiscriminado de los antibióticos, ya sea administrados a humanos o a animales (13). Las bacterias transmiten la resistencia a las siguientes generaciones, de esta manera se ejerce una presión de selección sobre aquellos microorganismos resistentes a los antibióticos, por lo cual, cada vez se hace más difícil el control de bacterias que producen graves enfermedades para el ser humano (14).

Lactobacillus lactis es una bacteria ácido-láctica que se ha estudiado por presentar características adecuadas como probiótico, presenta un antagonismo a bacterias Gram-positivas y Gram-negativas (15). Una de sus principales características es la producción de nisina (bacteriocina), que le permite reducir las poblaciones bacterianas patógenas presentes en alimentos (16). Se encuentra en gran variedad de ambientes, desde el tracto gastrointestinal hasta productos fermentados como el yogurt (17). De igual manera, reduce el $\mathrm{pH}$ del medio y produce ácidos orgánicos que también afectan a microorganismos no deseados (18).

El objetivo de la presente investigación fue determinar los parámetros de la cinética de crecimiento de Lactobacillus lactis y su efecto probiótico sobre Escherichia coli, Salmonella typhimurium, Clostridium perfringens $\mathrm{y}$ Staphylococcus aureus.

\section{METODOLOGÍA}

Se usaron cepas de Lactobacillus lactis ATCC® 11454, Escherichia coli ATCC $® 25922$, Salmonella typhimurium ATCC® 25241, Clostridium perfringens ATCC® 13124 y Staphylococcus aureus ATCC® 25923, que fueron reconstituidas de acuerdo con la casa fabricante. Las cepas fueron repicadas, para su conservación, en medio sólido y líquido cada 5 y 8 días respectivamente. Para la cepa láctica se usó medios MRS (sólido y líquido), mientras que las bacterias patógenas se reconstituyeron en caldo BHI como medio líquido y como medio sólido agar MacConkey para E. coli, agar XLD para S. typhimurium, agar SPS para C. perfringens y agar BD BairdParker para S. aureus. Todas las bacterias fueron incubadas a $37^{\circ} \mathrm{C}$ durante $24 \mathrm{~h}$ y se refrigeraron a $4^{\circ} \mathrm{C}$ para su posterior uso.

El inóculo de L. lactis se obtuvo de la siguiente manera: en un Erlenmeyer con $40 \mathrm{ml}$ de caldo MRS estéril, se depositó una alícuota de la cepa láctica y se incubó a $35^{\circ} \mathrm{C}$ por $24 \mathrm{~h}$ (preparado a). Al terminar el periodo de incubación, se tomaron $4 \mathrm{ml}$ del preparado a y se depositaron en un nuevo Erlenmeyer con otros $40 \mathrm{ml}$ de caldo MRS estéril al cual denominamos preparado $b$, este se incubó a la misma temperatura y tiempo de la primera incubación.

El ajuste del inóculo fue realizado a $10 \% \mathrm{v} / \mathrm{v}$ mediante la metodología de Crueger y Crueger (19), para ello se prepararon $90 \mathrm{ml}$ de caldo MRS estéril y se añadieron $10 \mathrm{ml}$ del preparado b. Después se realizó el conteo de bacterias por $\mathrm{ml}$. Para el conteo se tomó $1 \mathrm{ml}$ y se realizó lectura directa en espectrofotómetro a $625 \mathrm{~nm}$. En los casos donde la población fue mayor a la establecida, se adicionó caldo estéril mediante cálculo de proporcionalidad, de la siguiente manera:

$\mathrm{M}_{1}=$ población $\mathrm{o}$ densidad celular que se debe ajustar.

$\mathrm{M}_{2}=0,125$ densidad óptica equivalente a 1,50 $x 10^{8} \mathrm{ufc} / \mathrm{ml}$. Densidad utilizada primera fermentación. 
$\mathrm{V}_{1}=1 \mathrm{ml}$ volumen proveniente del inóculo total $(10 / 90) 1$

$\mathrm{X}_{1}=$ cantidad que contiene $\mathrm{M}_{2}$.

$\mathrm{V}_{2}=$ lo que se agrega a $1 \mathrm{ml}$ para ajustar a 1,50 $x 10^{8} \mathrm{ufc} / \mathrm{ml}$.

$\mathrm{V}_{3}=100 \mathrm{ml}$ cantidad total del inóculo.

$\mathrm{X}_{2}=$ cantidad de caldo MRS comercial estéril que se agrega $\mathrm{a}_{3}$ para ajustar la población al valor de $\mathrm{M}_{2}$.

Encontramos entonces $\mathrm{X}_{1}$

$\mathrm{M}_{1}-----\mathrm{M}_{2}$

$$
X_{1}=\left(\frac{M_{2} * V_{1}}{M_{1}}\right)
$$

Se evaluó el crecimiento in vitro de la cepa láctica, bajo tres concentraciones de sales biliares $(0,5,1$ y $2 \%$ ) y dos de bilis bovina (1 y 1,5\%). Para cada concentración se cultivó la bacteria en MRS por $24 \mathrm{~h}$, luego se tomaron alícuotas del cultivo y se depositaron en tubo de ensayo con caldo MRS en las concentraciones requeridas. De los tubos, se tomaron muestras, que se trasladaron a cajas de Petri con MRS y azul de anilina y se incubaron a $32^{\circ} \mathrm{C}$ por $48 \mathrm{~h}$. Al terminar la incubación se realizó recuento de bacterias para cada muestra.

También, se determinó producción de gas para $L$. lactis de acuerdo con la metodología de Dahl et al. (20) y la reacción de catalasa de acuerdo con Cai et al. (21). Se evaluó el crecimiento de L. lactis a tres $\mathrm{pH}(2,5,4,5$ y 7$)$ en medio MRS comercial durante un periodo de $3 \mathrm{~h}$ a $32^{\circ} \mathrm{C}$. El $\mathrm{pH}$ durante la prueba fue ajustado con ácido tartárico.

Se determinó la susceptibilidad de las bacterias a los antibióticos Dicloxacilina (DCX $1 \mu \mathrm{g}$ ), Cefepime (FEP $30 \mu \mathrm{g}$ ), Cefalotina (KF $30 \mu \mathrm{g}$ ), Ciprofloxacina (CIP $5 \mu \mathrm{g})$, Gentamicina (CN 10 $\mu \mathrm{g})$ y Penicilina (P10 IU) mediante la técnica de Kirby-Bauer (22).

Se evaluó el efecto de inhibición de L. lactis en las bacterias patógenas con la metodología propuesta por Tagg y McGiven (23) ajustada a las condiciones de nuestro laboratorio, de la siguiente manera: se tomó una alícuota de cada bacteria patógena y fue ajustada a 0,5 en escala MacFarland, luego se depositó en cajas de Petri con agar Müeller-Hinton; sobre las cajas de Petri se colocaron discos de agar impregnados con L. lactis en concentraciones de 25, 50 y $100 \mu \mathrm{l}$. Finalmente, las cajas fueron incubadas a $32^{\circ} \mathrm{C}$ por $24 \mathrm{~h}$. Transcurrido este tiempo, se midió la distancia del halo producido por la bacteria láctica, se utilizó como criterio de inhibición halos superiores a $2 \mathrm{~mm}(9)$.

También, se determinó el efecto de inhibición del sobrenadante de L. lactis sobre las bacterias patógenas con la metodología de Kirby-Bauer (22) modificada. Para obtener el sobrenadante se ajustó la bacteria láctica a 1 en escala MacFarland, se tomaron muestras de 1,5 ml y fueron depositadas en tubos Eppendorf para centrifugar a $15000 \mathrm{rpm} \mathrm{y} 4^{\circ} \mathrm{C}$ por $15 \mathrm{~min}$. Se separó el sobrenadante y se dividió en dos partes, la primera sin filtrar y la segunda filtrada con papel filtro de $0,25 \mu \mathrm{m}$. Para la prueba se utilizaron dos metodologías, la primera usando discos de papel pads, y la segunda cilindros plásticos (24), sobre cada uno (discos y cilindros) se depositaron concentraciones de 50, 75 y $100 \mu 1$ mediante micropipeta y se llevaron a incubación a $32^{\circ} \mathrm{C}$ por $24 \mathrm{~h}$.

Se evaluó la cinética de fermentación en un medio no comercial PRO (25) y el medio comercial MRS. El procedimiento fue el mismo para ambos medios: en un Erlenmeyer se colocaron $60 \mathrm{ml}$ de inóculo de L. lactis y $540 \mathrm{ml}$ de medio, se incubó a $32^{\circ} \mathrm{C}$ y $100 \mathrm{rpm}$, sin control del pH. Se midió el crecimiento cada $2 \mathrm{~h} 24 \mathrm{~min}$ por $24 \mathrm{~h}$ (ufc/ml). Para ello se realizó dilución de $1 \mathrm{ml}$ de muestra en $9 \mathrm{ml}$ de agua peptonada al $0,1 \%$, se realizaron diluciones decimales y se transfirieron a caja de Petri con medio MRS y azul de anilina $(0,1 \mathrm{ml})$ para siembra en superficie, las cajas se incubaron a $32^{\circ} \mathrm{C}$ por $24 \mathrm{~h}$, solo se tuvieron en cuenta los conteos entre 30 y 300 colonias, el número de colonias se multiplicó por el inverso de la dilución y 10 para obtener ufc/ml (26). 
Durante la cinética de fermentación, únicamente en el medio MRS, se realizaron mediciones de $\mathrm{pH}$, con un pHmetro digital; azúcar total, con el método de Dubois et al. (27); acidez mediante titulación con hidróxido de sodio (1N) (28) y proteína con el método de Lowry et al. (29). Los datos de crecimiento en el medio MRS se determinaron mediante la metodología de Crueger y Crueger (19) y Rodríguez-León et al. (30). Se estableció la velocidad máxima de crecimiento a través de la siguiente ecuación:

$$
v \max =\frac{d \operatorname{Ln} X}{d t}
$$

y el tiempo de duplicación celular (td), se determinó teniendo en cuenta la ecuación:

$$
t d=\frac{\operatorname{Ln} 2}{v \max }
$$

Se determinó la viabilidad de L. lactis a dos temperaturas $\left(38\right.$ y $\left.45^{\circ} \mathrm{C}\right)$. La cepa fue evaluada hasta las $14 \mathrm{~h} 24 \mathrm{~min}$ (se tuvo en cuenta el tiempo necesario para llegar a la fase exponencial en el medio MRS). Se usó el procedimiento descrito por Crueger y Crueger (19), con ajuste del inóculo a 0,125 en escala de McFarland. Luego se hicieron diluciones de $10^{-1}$ hasta $10^{-12}$ con agua peptonada y se sembró en cajas de Petri con azul de anilina, enseguida se inició en la dilución $10^{-8}$ hasta $10^{-12}$ a $37^{\circ} \mathrm{C}$ y $48 \mathrm{~h}$, finalmente se determinó el número de ufc/ml.

En el sobrenadante de L. lactis se determinó el contenido de péptidos mediante espectrofotometría de alta densidad (HPLC), se tomó una alícuota de $25 \mathrm{ml}$ y fue centrifugada a $18000 \mathrm{rpm}$ por $30 \mathrm{~min}$ a $4^{\circ} \mathrm{C}$; luego se filtraron 2 $\mathrm{ml}$ en jeringa de filtrar $(0,25$ micras $)$ y finalmente fue llevado a lectura en el espectrofotómetro a 650 nm para obtención de los resultados.

Los procedimientos estadísticos se realizaron en el paquete SAS 9.1 (31). Se evaluó el crecimiento de los medios MRS y PRO durante la cinética de fermentación con el usó de medidas repetidas en el tiempo (PROC MIXED de SAS).

$\mathrm{y}_{\mathrm{ijk}}=\mu+\mathrm{A}_{\mathrm{i}}+\mathrm{B}_{\mathrm{k}}+\left(\mathrm{A}^{*} \mathrm{~B}\right)_{\mathrm{ik}}+\varepsilon_{\mathrm{ij}}+\varepsilon_{\mathrm{ijk}}$ donde:

$\mathrm{y}_{\mathrm{ijk}} \quad$ : observación ijk.

$\mu \quad$ : media general.

$\mathrm{A}_{\mathrm{i}} \quad$ : efecto de tratamiento.

$B_{k} \quad$ : efecto del tiempo.

$\left(A^{*} B\right)_{i k}$ : efecto de interacción entre los tratamientos i y el tiempo $\mathrm{k}$.

$\varepsilon_{\mathrm{ij}} \quad$ : error aleatorio y covarianza entre medidas repetidas.

: error aleatorio.

Los resultados obtenidos en la prueba de inhibición con el sobrenadante (discos y cilindros plásticos) fueron correlacionados (Correlación de Pearson, PROC CORR deSAS), y los parámetros $\mathrm{pH}$, acidez, azúcar y ácido láctico de la cinética de fermentación se relacionaron con el crecimiento ( $\mathrm{ufc} / \mathrm{ml})$, mediante regresión lineal simple (PROC REG de SAS).

$Y=\beta_{0}+\beta_{x 1}+\mathrm{e}$

donde:

y: variable a evaluar.

$\beta_{0}$ : intercepto.

$\beta_{1 \mathrm{x}}$ : pendiente.

e: error.

\section{RESULTADOS}

Las pruebas de bilis bovina mostraron que $L$. lactis no crece a concentraciones de $1 \%$ pero sí a $1,2 \%$ con un valor promedio de $1,2 \times 10^{9} \mathrm{ufc} /$ $\mathrm{ml}$. Las pruebas de sales biliares mostraron crecimientos únicamente a $0,5 \%$ con un valor promedio de 4,1 x $10^{9} \mathrm{ufc} / \mathrm{ml}$. Las pruebas de catalasa y gas fueron negativas. El crecimiento a diferentes $\mathrm{pH}$ demostró que la cepa láctica presenta viabilidad al final de las tres horas, con valores de $1,1 \times 10^{11}, 2,1 \times 10^{10}$ y $1,0 \times 10^{10} \mathrm{ufc} / \mathrm{ml}$ para $\mathrm{pH} 2,5,4,5$ y 7 respectivamente.

Los resultados de la prueba de susceptibilidad a los antibióticos se observan en la Tabla 1 y en la Figura 1. La cepa láctica presentó resistencia a la Dicloxacilina y Cefalotina; C. perfringens, a Dicloxacilina y Penicilina; $S$. typhimurium y $E$. coli, a Cefalotina; y $S$. aureus, a Dicloxacilina. 
Henry Jurado-Gámez, Verónica Jarrín-Jarrín

Tabla 1. Susceptibilidad de las bacterias a los antibióticos evaluados.

\begin{tabular}{|c|c|c|c|c|c|c|c|c|c|c|c|c|}
\hline \multirow{4}{*}{\begin{tabular}{l}
\multicolumn{1}{c}{$\begin{array}{c}\text { Cepas de } \\
\text { estudio ATCC }\end{array}$} \\
L. lactis
\end{tabular}} & \multicolumn{12}{|c|}{ Diámetros del halo de inhibición (mm) } \\
\hline & \multicolumn{12}{|c|}{ Antibióticos } \\
\hline & \multicolumn{2}{|c|}{ DCX 1} & \multicolumn{2}{|c|}{$\begin{array}{c}\text { FEP } \\
30 \\
\end{array}$} & \multicolumn{2}{|c|}{ KF 30} & \multicolumn{2}{|c|}{ CIP 5} & \multicolumn{2}{|c|}{ CN 10} & \multicolumn{2}{|c|}{ P 10} \\
\hline & 8 & $\mathbf{R}$ & 10 & $\mathrm{R}$ & 8 & $\mathbf{R}$ & 46 & S & 26 & S & 35 & $S$ \\
\hline \multicolumn{13}{|c|}{ Bacterias patógenas } \\
\hline C. perfringens & 6 & $\mathbf{R}$ & 10 & $\mathbf{R}$ & 30 & $S$ & NA & - & NA & - & 8 & $\mathbf{R}$ \\
\hline S. typhimurium & NA & - & 32 & $S$ & 12 & $\mathbf{R}$ & 45 & $S$ & 23 & $S$ & NA & - \\
\hline E. coli & NA & - & 30 & $S$ & 10 & $\mathbf{R}$ & 42 & $S$ & 22 & $S$ & NA & - \\
\hline S. aureus & 6 & $\mathbf{R}$ & 20 & $S$ & 45 & $S$ & NA & - & NA & - & 20 & $S$ \\
\hline
\end{tabular}

DCX: Dicloxacilina; FEP: Cefepime; KF: Cefalotina; CIP: Ciprofloxacina; CN: Gentamicina; P 10: Penicilina; NA: No aplica; S: sensible; R: resistente. (Clinical and Laboratory Standards Institute, 2011; EUCAST, European Committee on Antimicrobial Susceptibility Testing, 2012).

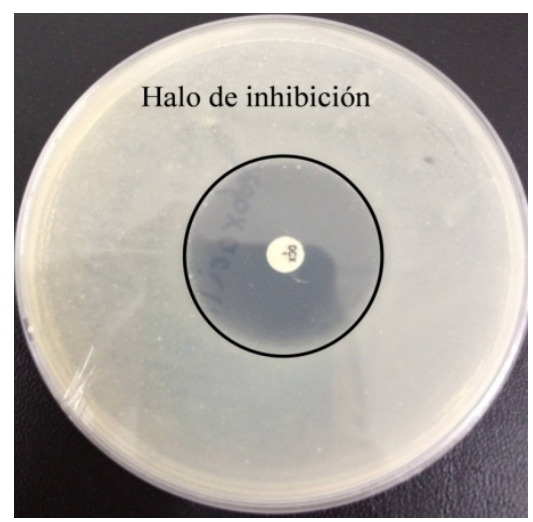

Figura 1. Halo de inhibición de Dicloxacilina sobre E. coli.

La prueba de inhibición de L. lactis mostró susceptibilidad de $C$. perfringens y $E$. coli en las tres concentraciones $(25,50$ y $100 \mu \mathrm{l}) ; \mathrm{S}$. tiphymurium a $100 \mu \mathrm{l}$; y . aureus no fue inhibida en ninguna de las concentraciones (Tabla 2 y Figura 2).
Tabla 2. Efecto de inhibición de L. lactis sobre las bacterias patógenas.

\begin{tabular}{lccc}
\hline \multicolumn{1}{c}{ CEPAS } & $\mathbf{2 5} \boldsymbol{\mu l}$ & $\mathbf{5 0} \boldsymbol{\mu l}$ & $\mathbf{1 0 0} \boldsymbol{\mu l}$ \\
\hline S. aureus & 0,833 & 0,667 & $\mathbf{1 , 1 6 7}$ \\
C. perfringens & $\mathbf{3 , 3 3 3}$ & $\mathbf{4 , 6 6 7}$ & $\mathbf{5 , 0 0 0}$ \\
S. tiphymurium & $\mathbf{1 , 6 6 7}$ & 1,500 & $\mathbf{2 , 0 0 0}$ \\
E. coli & $\mathbf{3 , 0 0 0}$ & $\mathbf{2 , 6 6 7}$ & $\mathbf{4 , 5 0 0}$ \\
\hline
\end{tabular}

Valores iguales o superiores a $2 \mathrm{~mm}$ muestran susceptibilidad (19). 

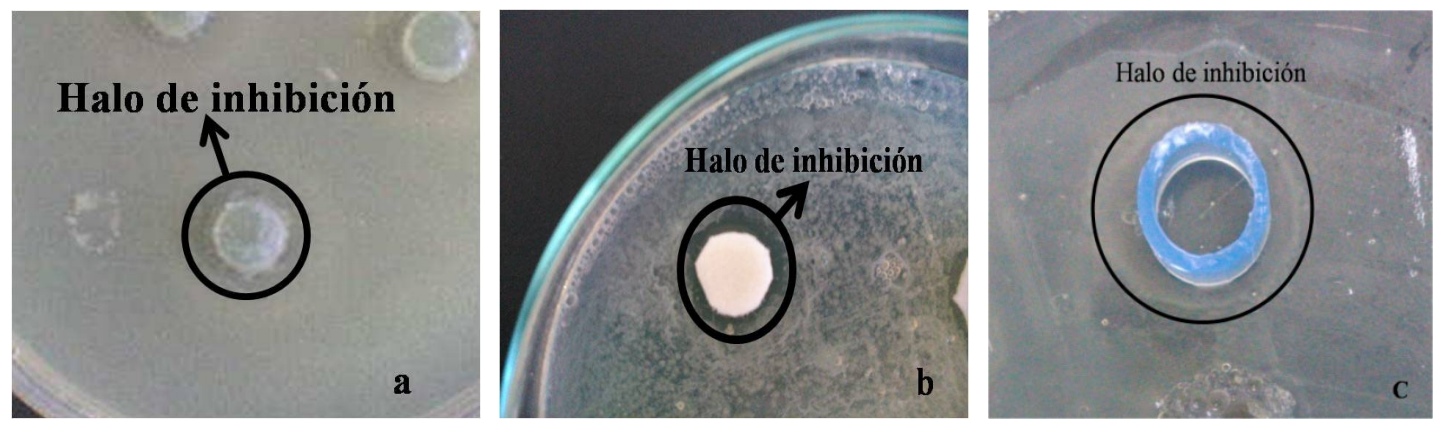

Figura 2. Halos de inhibición de L. lactis y su sobrenadante sobre las bacterias patógenas. a: disco de gel con L. lactis; b: sobrenadante en sensidisco; c: sobrenadante en cilindro.

La evaluación mediante el sensidisco impregnado de sobrenadante de L. lactis inhibió a S. aureus en las tres concentraciones (50,75 y $100 \mu \mathrm{l}) ; C$. perfringens y $S$. tiphymurium a $50 \mu \mathrm{l} ; \mathrm{y}$ E. coli no fue inhibida (Tabla 3 y Figura 2). Mediante la metodología de cilindros se encontró que $S$. aureus era susceptible a todas las concentraciones y S. tiphymurium a 100 y $50 \mu$ l para el sobrenadante filtrado y sin filtrar respectivamente.

Tabla 3. Halos de inhibición de los discos y cilindros impregnados con sobrenadante de L. lactis sobre las bacterias patógenas.

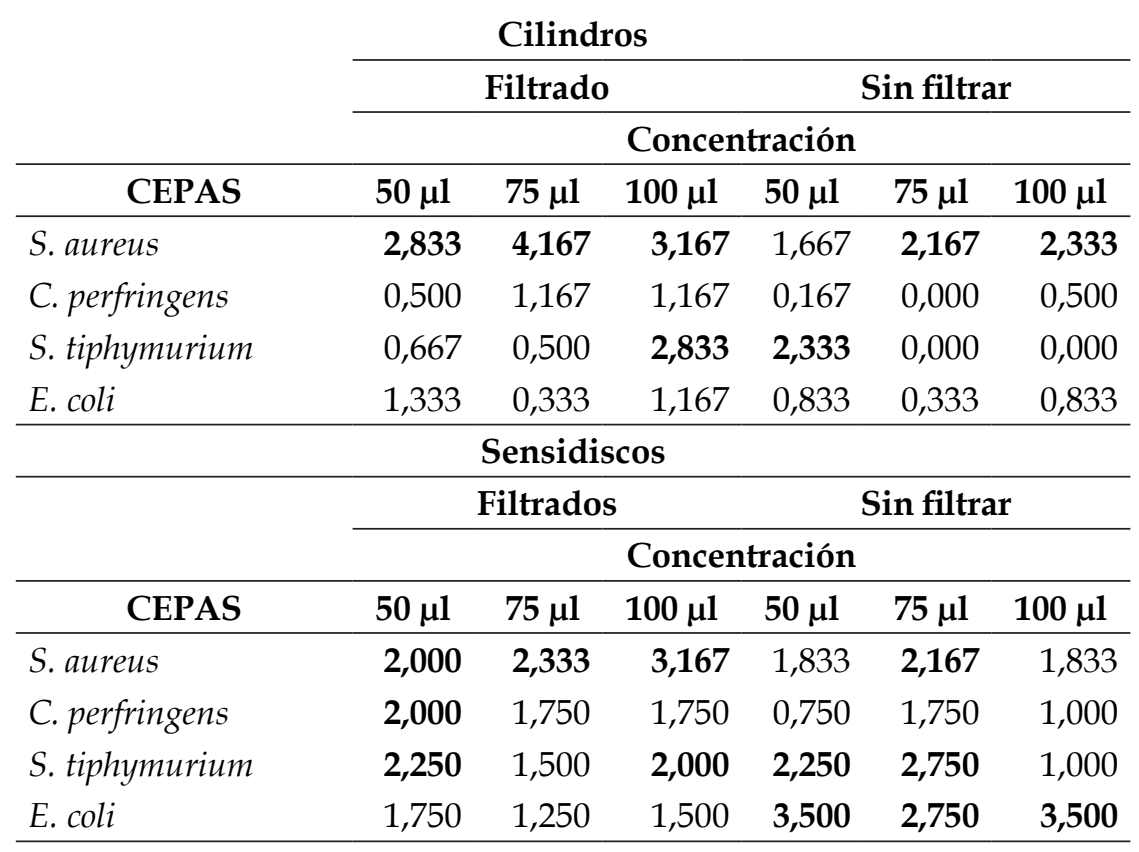

No se encontró correlación entre los dos métodos usados (sensidisco, cilindro) para evaluar la inhibición del sobrenadante (Corr: 0,2658; p-value: 0,2092).
Los resultados de la cinética de fermentación, el $\mathrm{pH}$, la acidez, el azúcar y proteína medidos durante el tiempo de fermentación se observan en la Figura 3. 


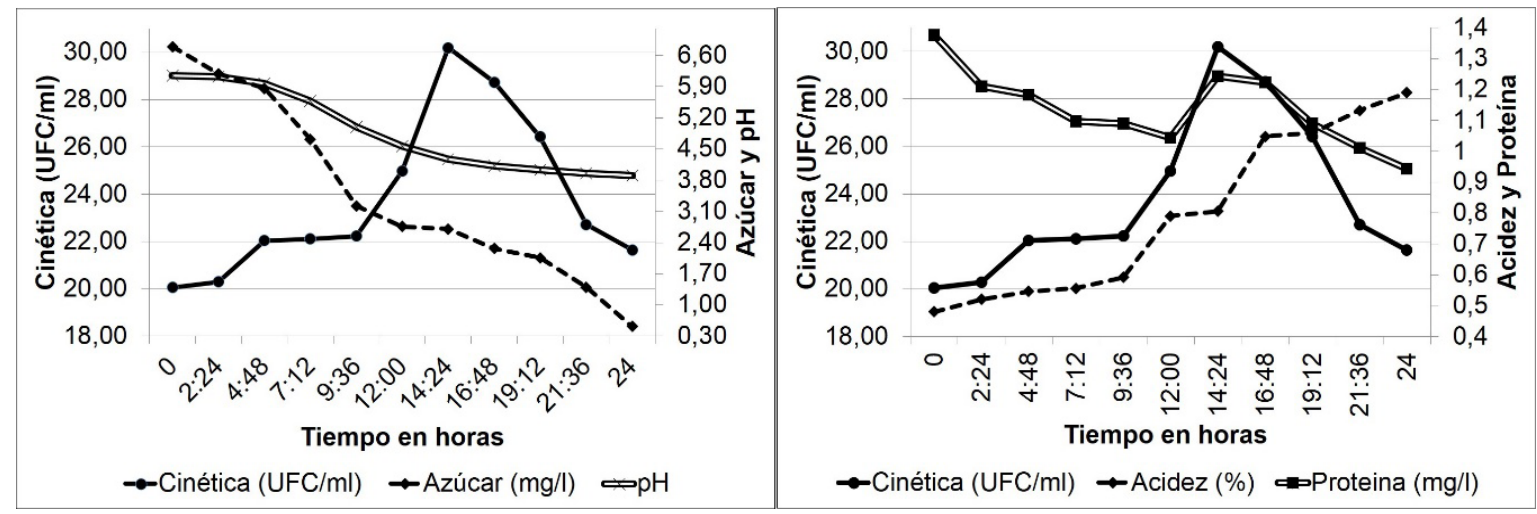

Figura 3. Cinética de fermentación, $\mathrm{pH}$, azúcar, acidez y proteína de L. lactis en medio MRS.

La fase exponencial de L. lactis se obtuvo a las $14 \mathrm{~h} 24 \mathrm{~min}$ con un crecimiento de $1,3 \times 10^{13}$ $\mathrm{ufc} / \mathrm{ml}$ en el medio MRS; durante este periodo se observó un valor de 4,26 en el $\mathrm{pH} ; 0,806 \%$ en la acidez, 1,24 mg/L de proteína y $2,70 \mathrm{mg} / \mathrm{L}$ de azúcar. El análisis de regresión mostró un valor de 0,0339 para acidez $\left(R^{2}: 0,794 ; p\right.$-value: $0,00437)$; -0,3834 para azúcar $\left(\mathrm{R}^{2}\right.$ : 0,530; $\mathrm{p}$-value: $0,03849)$; -0,1936 para $\mathrm{pH}\left(\mathrm{R}^{2}: 0,735\right.$; $\mathrm{p}$-value: $0,0084) ;$ y $-0,0050$ para proteína $\left(\mathrm{R}^{2}: 0,023\right.$; p-value: 0,741$)$. Los datos de la cinética de crecimiento se pueden observar en la Tabla 4.

Tabla 4. Datos de la cinética de crecimiento.

\begin{tabular}{rc}
\hline \multicolumn{2}{c}{ Medio MRS } \\
\hline Fase Lat & 0 \\
Vel. Esp. Crec. $\left(\boldsymbol{\mu} \mathbf{~ h}^{-1}\right)$ & 14,48 \\
Fin Fase Log (h) & $14: 24$ \\
Tiem. Dupl. (min) & 2,87 \\
Incr. Cel. Total & $2,50 \mathrm{E}+09$ \\
Incr. Cel. Final & $3,30 \mathrm{E}+13$ \\
\% Azúc. Cons. Total $(\mathbf{g} / \mathbf{L})$ & $92,41 \%$ \\
\% Azúc. Cons. Fin. Total $(\mathbf{g} / \mathbf{L})$ & $60,26 \%$ \\
\% Prot. Cons. Total $(\mathbf{g} / \mathbf{l})$ & $31,48 \%$ \\
\% Prot. Cons. Fase Log $(\mathbf{g} / \mathbf{l})$ & $9,69 \%$ \\
$\mathbf{r}^{2}=$ & 0,772 \\
\hline
\end{tabular}

La prueba de viabilidad de la bacteria láctica a dos temperaturas mostró crecimientos adecuados con valores de $1,7 \times 10^{13}$ y $1,4 \times 10^{13}$ ufc/ $\mathrm{ml}$ para 45 y $38^{\circ} \mathrm{C}$ respectivamente, a las 14 h $24 \mathrm{~min}$ (fase exponencial).
El análisis del sobrenadante de L. lactis por HPLC mostró una cadena de péptidos VAL-TIR-VAL en el tiempo 11,842 con una concentración de 0,66 $\mathrm{mg} / \mathrm{ml}$ de muestra (figuras 4 y 5 ; tablas 5 y 6 ). 


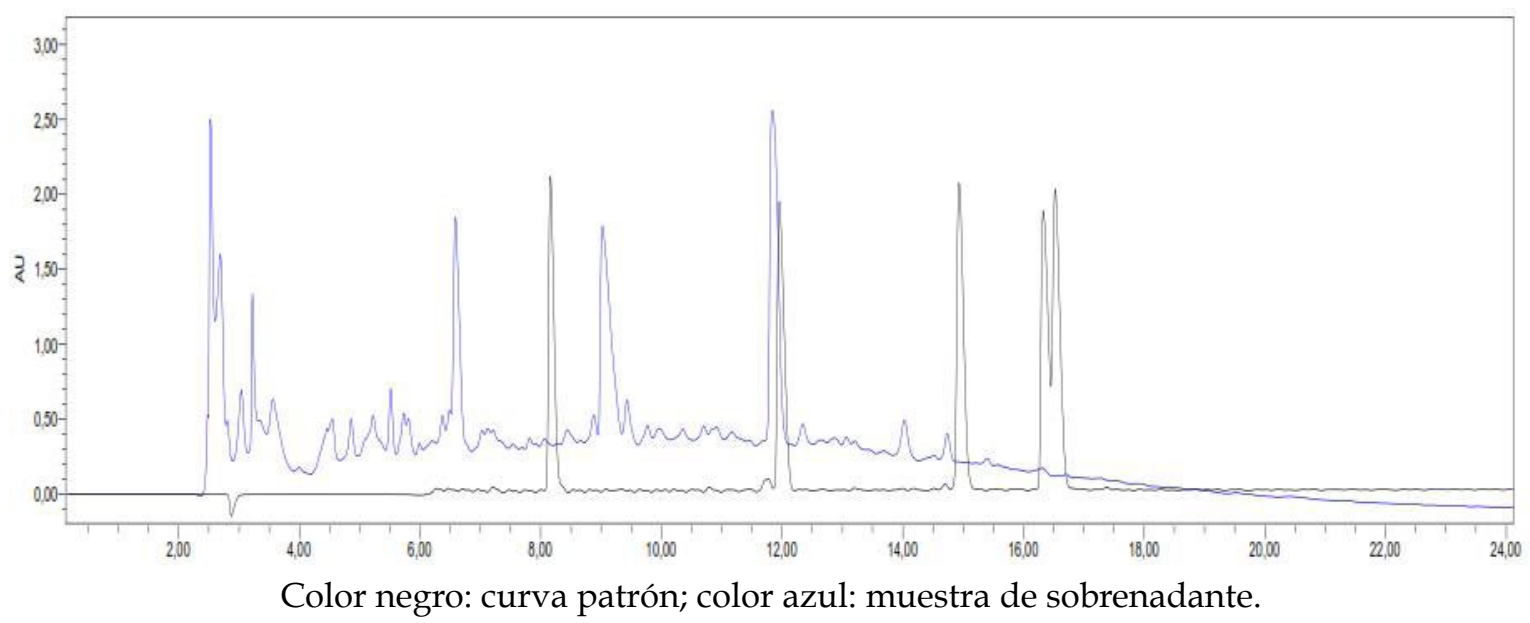

Figura 4. Cromatograma del sobrenadante de Lactobacillus lactis.

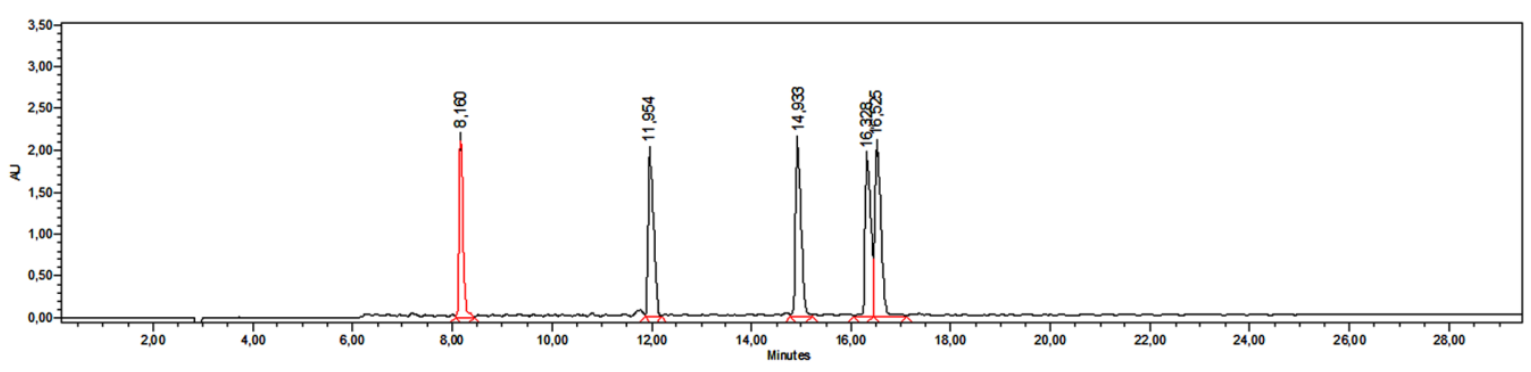

Figura 5. Cromatograma de péptidos estándar.

Tabla 5. Péptidos estándar.

\begin{tabular}{ccccc}
\hline No. Pico & Nombre & $\begin{array}{c}\text { Tiempo de } \\
\text { retención }\end{array}$ & Área & $\begin{array}{c}\text { Cantidad relativa } \\
\%\end{array}$ \\
\hline 1 & GLY-TYR & 8,16 & 13536607 & 17,32 \\
2 & VAL-TYR-VAL & 11,954 & 15409159 & 19,71 \\
3 & Metionine Enkefalin Acetate & 14,933 & 16289583 & 20,84 \\
4 & Leucine Enkefalin & 16,328 & 15163721 & 19,4 \\
5 & Angiotensin II Acetate & 16,525 & 17776696 & 22,74 \\
\hline
\end{tabular}


Henry Jurado-Gámez, Verónica Jarrín-Jarrín

Tabla 6. Tiempos de retención encontrados en la muestra a través de HPLC.

\begin{tabular}{ccc}
\hline No. Pico & Tiempo de retención & Cantidad relativa en \% promedio \\
\hline 1 & 2,526 & 6,74 \\
2 & 2,695 & 5,69 \\
3 & 3,039 & 3,43 \\
4 & 3,567 & 2,99 \\
5 & 5,514 & 2,31 \\
6 & 6,585 & 12,38 \\
7 & 9,021 & 22,48 \\
8 & $\mathbf{1 1 , 8 4 2}$ & $\mathbf{3 0 , 9 2}$ \\
9 & 12,343 & 0,99 \\
10 & 14,026 & 2,52 \\
11 & 14,737 & 1,46 \\
12 & 15,398 & 0,33 \\
\hline
\end{tabular}

\section{DISCUSIÓN}

Para determinar la viabilidad de cepas probióticas es necesario realizar evaluación de los microorganismos en las condiciones gastrointestinales del animal huésped. Entre los factores que tienen preponderancia para la sobrevivencia de la cepa está su resistencia a la bilis y las sales biliares secretadas por el hígado. Un crecimiento superior a $10^{9} \mathrm{ufc} /$ $\mathrm{ml}$ es considerado adecuado para una buena colonización del tracto digestivo (25). De acuerdo con lo anterior, la cepa evaluada demostró apropiados crecimientos para bilis en condiciones in vitro a la concentración más baja. A pesar de no observarse crecimiento en las concentraciones más altas ( 1 y $2 \%$ ), la cepa láctica en condiciones gastrointestinales no se verá expuesta a niveles tan elevados, por lo que los resultados encontrados muestran viabilidad de la cepa. Kumer et al. (32) encontraron que las cepas lácticas presentan tolerancia a las sales biliares, además disminuyen el colesterol en el organismo huésped. Iguales resultados fueron encontrados por Hamon et al. (33) cuando evaluaron a $L$. plantarum. Las sales biliares producen estrés en los microorganismos colonizadores del sistema digestivo. Sin embargo, las bacterias generan respuestas fisiológicas como protección celular, generalmente modifican la membrana celular que les permiten tolerar estos cambios y adaptarse al medio gastrointestinal (34).

Las bacterias ácido-lácticas se caracterizan por ser catalasa negativa, característica observada en la presente investigación (35). La cepa láctica, al no producir gas, evita en el huésped problemas gastrointestinales como el timpanismo (24).

La evaluación a diferentes $\mathrm{pH}$ simula el tránsito de la cepa por el tracto gastrointestinal, los diferentes segmentos del tracto digestivo son afectados por variación en el $\mathrm{pH}$ del medio. Así, en el estómago se observan $\mathrm{pH}$ cercanos a 2 en especies domésticas, mientras que, en el intestino delgado, entre 5 a 7 como consecuencia de la secreción del hígado (36). Los crecimientos encontrados en la investigación muestran la viabilidad de la cepa láctica a estas condiciones, con poblaciones adecuadas para colonizar el tracto digestivo. Los Lactobacillus son considerados acidúricos debido a que crecen a $\mathrm{pH}$ inferiores a 5 (37). Su capacidad de resistencia a estos valores se debe a mecanismos 
celulares que le permiten mantener el $\mathrm{pH}$ interno cerca de la neutralidad como la bomba de extracción de protones (38). Otro de los factores que argumentan este comportamiento se debe a su capacidad de producir ácidos orgánicos como resultado de la fermentación de carbohidratos, que con la acumulación disminuyen el $\mathrm{pH}$ del medio (39).

Determinar el efecto de inhibición de una bacteria láctica sobre cepas patógenas es importante para establecer su potencial probiótico. Los mecanismos que usa para generar antagonismo con otros microorganismos son varios, entre los que se encuentran producción de biocinas, reducción del $\mathrm{pH}$, producción de ácidos orgánicos y producción de peróxido (40). Los resultados muestran que la cepa láctica inhibe a C. perfringens y E. coli en condiciones in vitro. Sankar et al. (41) encontraron susceptibilidad de E. coli y $S$. aureus por bacteriocinas producidas por L. plantarum, mientras que Rajaram et al. (42) encontraron que L. lactis inhibió a E. coli y $S$. aureus. A pesar de que las cepas $S$. aureus y $S$. tiphymurium fueron resistentes a la cepa láctica, el sobrenadante inhibió a $S$. aureus, mostrando un efecto de la cepa en la bacteria patógena. Los resultados encontrados indican que L. lactis posee características adecuadas para el control de tres de las bacterias patógenas evaluadas. Los resultados encontrados entre metodologías para evaluar al sobrenadante fueron similares a otros estudios realizados por el autor (24). La metodología de cilindros debe continuar en evaluación con el fin de obtener suficiente información que permita determinar resultados válidos para su estimación.

El crecimiento durante la fase exponencial demostró eficiencia de la cepa láctica en la producción de inóculos, ya sea para su producción a nivel industrial, como para su crecimiento a nivel gastrointestinal. El pH alcanzado durante la fase exponencial es cercano a 4, sin embargo, se observa que el $\mathrm{pH}$ del medio descendió hasta un valor de 3,8 demostrando el efecto de reducción del medio producido por la cepa láctica. Este descenso es importante, ya que permite inhibir el crecimiento de otras bacterias (43). La producción de ácido láctico está estrechamente relacionada con la reducción del $\mathrm{pH}$; entre los productos de la fermentación de L. lactis se encuentra el ácido láctico y con ello el aumento de la acidez del medio (44). El porcentaje de consumo de azúcar fue de $92,41 \%$ durante todo el periodo de fermentación, esto demuestra el buen uso del nutriente por parte de la cepa láctica $y$, de manera indirecta, la producción de ácidos orgánicos como productos de la fermentación (45).

La temperatura interna de los animales homeotermos oscila entre los 37 y $41^{\circ} \mathrm{C}$, de esta manera con la temperatura de $38^{\circ} \mathrm{C}$ se evalúa la viabilidad de la cepa a condiciones de temperatura corporal; como los resultados del crecimiento fueron altos, se puede argumentar que la cepa podría resistir la temperatura interna de algunos animales domésticos. La temperatura de $45^{\circ} \mathrm{C}$ evalúa la viabilidad de la cepa a tratamientos térmicos realizados en los alimentos balanceados que tiene como fin su peletización. Sin embargo, se debe realizar otro tipo de pruebas para determinar si la cepa resiste a los procesos industriales de obtención de alimentos balanceados.

Se concluye que L. lactis presenta parámetros de crecimiento adecuados en condiciones in vitro que simulan el ambiente del sistema digestivo, puede inhibir el crecimiento de E. coli, C. perfringens y $S$. aureus, pero no a $S$. typhimurium en condiciones in vitro y tolera temperaturas internas de su huésped. 


\section{REFERENCIAS}

1. Cueto-Vigil MC, Acuña-Monsalve $Y$, Valenzuela-Riaño J. Evaluación in vitro del potencial probiótico de bacterias ácido lácticas aisladas de suero costeño. Actual Biol 2010; 32(93):129-138.

2. Rajaram G, Manivasagan $P$, Thilagavathi $B$, Saravanakumar A. Purification and characterization of a Bacteriocin produced by Lactobacillus lactis isolated from marine environment. Advance Journal of Food Science and Technology 2010; 2(2):138-144.

3. Mohankumar A, Murugalatha N. Characterization and Antibacterial Activity of Bactereocin Producing Lactobacillus Isolated from Raw Cattle Milk Sample. Intern. J. Biol. 2011; 3(3):128-143.

4. González-Olivares LG, Jiménez-Guzmán J, Cruz-Guerrero A, Rodríguez-Serrano G, Gómez-Ruiz L, García-Garibay M. Liberación de péptidos bioactivos por bacterias lácticas en leches fermentadas comerciales. Revista Mexicana de Ingeniería Química 2011; 10(2):179-188.

5. Marguet ER, Vallejo M, Sierralta-Chichisola V, Quispe JL. Actividad antagonista de bacterias lácticas aisladas del medio marino contra cepas de Listeria. Acta Bioquím. Clín. Latinoam. 2011; 45(2):305310.

6. Pérez $M$, Laurencio $M$, Rondón AJ, Milian G, Bocourt R, Arteaga F. Actividad antimicrobiana de una mezcla probiótica de exclusión competitiva y su estabilidad en el tiempo. Rev. Salud Anim. 2011; 33(3):147-153.

7. Karthikeyan $\mathrm{V}$, Santosh SW. Isolation and parcial characterization of bacteriocin produced from Lactobacillus plantarum. Afr. J. Microbiol. Res. 2009; 3(5):233-239.

8. Dal Bello B, Cocolin L, Zeppa G, Field D, Cotter PD, Hill C. Technological characterization of bacteriocin producing Lactococucus lactis strains employed to control Listeria monocytogenes in Cottage cheese. Intern. J. Food Microbiol. 2012; 153:58-65.

9. Estrada A, Gutiérrez L, Montoya O. Evaluación in vitro del efecto bactericida de cepas nativas de Lactobacillus sp. contra Salmonella sp. y Escherichia coli. Rev. Fac. Nal. Agr. Medellín 2005; 58(1):2601-2609.

10. Cajarville C, Brambillasca S, Zunino P. Utilización de prebióticos en monogástricos: aspectos fisiológicos y productivos relacionados al uso de subproductos de agroindustrias y de pasturas en lechones. Revista Porcicultura Iberoamericana 2011; 1-11.

11. Sánchez L, Vichi J, Llanes M, Castro E, Soler DM, Espinosa I, Kociubinski GL, Ferreira CL. Aislamiento y caracterización in vitro de cepas de Lactobacillus ssp. como candidato a probióticas. Rev. Salud Anim. 2011; 33(3):154-160.

12. Mathur S, Singh R. Antibiotic resistance in food lactic acid bacteria - a review. Intern. J. Food Microbiol. 2005; 105(3):281-295.

13. Cabrera CE, Gómez RF, Zúñiga AE. La resistencia de bacterias a antibióticos, antisépticos y desinfectantes una manifestación de los mecanismos de supervivencia y adaptación. Colombia Médica 2007; 38(2):149-158.

14. Junod T, López-Martín J, Gädicke P. Estudio de susceptibilidad antimicrobiana de Salmonella enterica en muestras de origen animal y alimentario. Rev. Med. Chile. 2013; 141:298-304.

15. Rajaram G, Manivasagan P, Gunasekaran U, Ramesh S, Ashokkumar S, Thilagavathi B, Saravanakumar A. Isolation, identification and characterization of bacteriocin from Lactobacillus lactis and its antimicrobial and cytotoxic properties. African Journal of Pharmacy and Pharmacology 2010; 4(12):895-902.

16. Dimov S, Ivanova P, Harizanova N. Genetics of bacteriocins biosynthesis by lactic acid bacteria. Biotechnology \& Biotechnological Equipment 2005; 19(Supl 2):4-10.

17. Eggimann B, Bachmann M. Purification and Partial characterization of an aminopeptidase from Lactobacillus lactis. Applied and Environmental Microbiology 1980; 40(5):876-882. 
18. Lade H, Chitannad M, Gyananath G, Kadam T. Studies On Some Properties of Bacteriocins Produced by Lactobacillus Species Isolated from Agro-Based Waste. The Intern. J. Microbiol. 2006; 2(1):1-4.

19. Crueger W, Crueger A. Biotecnología: Manual de Microbiología Industrial. 3 ed. España: Ed. Acribia; 1993.

20. Dahl T, Midden W, Hartman P. Killing of Gram-negative and Gram-positive Bacteria by Pure Singlet Oxygen. J Bacteriol 1989; 171(4):2188-2194.

21. Cai Y, Suyanandana P, Saman P, Benno Y. 1999. Classification and characterization of lactic acid bacteria isolated from the intestines of common carp and freshwater prawns. J Gen Appl Microbiol 1999; 45(4):177-184.

22. Bauer A, Kirby J, Sherris T. Antibiotic susceptibility testing by a standardized single disk method. Am. J. Clin. Pathol. 1966; 36:493-496.

23. Tagg J, McGiven A. 1971. Assay system for Bacteriocins. Appl. Environ. Microb. 1971; 21 (5):943.

24. Jurado-Gámez H, Calpa-Yama F, Chaspuengal-Tulcán A. Determinación in vitro de la acción probiótica de Lactobacillus plantarum sobre Yersinia pseudotuberculosis aislada de Cavia porcellus. Rev. Fac. Med. Vet. Zoot. 2014; 61(3):241-257.

25. Ramírez M. Actividad inhibitoria de cepas de bacterias acido lácticas frente a bacterias patógenas y deterioradoras de alimentos [tesis]. Pachuca de Soto, México: Universidad Autónoma del Estado de Hidalgo; 2005.

26. LANARA, Laboratorio de Referencia Animal. Métodos analíticos oficiais para controle de produtos de origem animal e seus ingredientes. Ii- Métodos físico e químicos. Mel. Ministério da Agricultura. Brasilia. 1981; 2(25):1-15.

27. Dubois M, Gilles K, Hamilton J, Rebers P, Smith F. Colorimetric method for determination of sugar and related substances. Anal Chem 1956; 28:350-356.

28. Negri L. El pH y la acidez de la leche; 2005. Disponible en: http://www.aprocal.com.ar/wp-content/ uploads/pH-y-acidez-en-leche2.pdf [consultado 2 de diciembre de 2013].

29. Lowry O, Rosebroug N, Far A, Randall R. Protein measurement with the folin phenol reagent. J. Biol. Chem. 1951; 193:265-75.

30. Rodríguez-León JA, Bueno G, Rodríguez DE, Delgado G, Serrano P, Brizuela MA. True and apparent yields and maintenance coefficient and their significance on fermentation kinetics. En: S. Roussos et al. (Eds.). New Horizons Biotechnology. Kluwer Academic Publishers; 2003. p. 163-172.

31. SAS Institute Inc. SAS/STAT ${ }^{\circledR} 9.1$ User's Guide. Cary, NC: SAS Institute Inc. ; 2004.

32. Kumar R, Grover S, Kumar-Batish V. Hypocholesterolaemic effect of dietary inclusion of two putative probiotic bile salt hydrolase-producing Lactobacillus plantarum strains in Sprague-Dawley rats. British Journal of Nutrition 2011; 105:561-573.

33. Hamon E, Horvatovich P, Izquierdo E, Bringel F, Marchioni E, Aoudé-Werner D, Ennahar S. Comparative proteomic analysis of Lactobacillus plantarum for the identification of key proteins in bile tolerance. BMC Microbiology 2011; 11:63.

34. Wu R, Sun Z, Wu J, Meng H, Zhang H. Effect of bile salts stress on protein synthesis of Lactobacillus casei Zhang revealed by 2 -dimensional gel electrophoresis. J. Dairy Sci. 2010; 93:3858-3868.

35. Adebayo-Tayo B, Onilude A. Screening of Lactic Acid Bacteria Strains Isotated from Some Nigerian Fermented Food for EPS production. World Applied Science Journal 2008; 4(5):741-747.

36. Teijón-Rivera JM, Garrido-Pertierra A, Blanco-Gaitán D, Villaverde-Gutiérrez C, Mendoza-Oltras C, Ramírez-Rodrigo J. Fundamentos de bioquímica metabólica. Segunda edición. Madrid: Editorial TÉBAR, S.L.; 2006.

37. Kandler O, Weiss N. Regular, Nosporing Gram-positive Rods. En: Sneath PH, Mair NS, Sharpe ME, Holt JG (Eds.). Bergey's manual of systematic bacteriology. Vol. 2. Baltimore (USA): Williams \& Wilkins; 1986 . p. 1208-1235. 
38. Piard J, Desmazeaud M. Inhibiting factors produced by lactic acid bacteria. Oxygen metabolites and catabolism end-products. Lait 1991; 71:525-541.

39. Broadbent J, Larsen R, Deibel V, Steele J. Physiological and Transcriptional Response of Lactobacillus casei ATCC 334 to Acid Stress. J. Bacteriol. 2010; 192(9):2445-2458.

40. Tambecar D, Buhutada S. Studies on Antimicrobial Activity and Characteristic of Bacteriocins produced by Lactobacillus Strains Isolated from Milk of Domestic Animals. The Internet Journal of Microbiology 2008; 8(2).

41. Sankar N, Priyanka D, Reddy P, Rajanikanth P, Kumar K, Indira M. Purification and Characterization of Bacteriocin Produced by Lactobacillus plantarum Isolated from Cow Milk. Inter. J. Microbiol. Res. 2012; 3(2):133-137.

42. Rajaram G, Manivasagan P, Thilagavathi B, Saravanakumar A. Purification and Characterization of a Bacteriocin Produced by Lactobacillus lactis Isolated from Marine Environment. Advance Journal of Food Science and Technology 2010; 2(2):138-144.

43. Allende A, Martínez B, Selma V, Gil M, Suárez J, Rodríguez A. Growth and bacteriocin production by lactic acid bacteria in vegetable broth and their effectiveness at reducing Listeria monocytogenes in vitro and in fresh-cut lettuce. J. Food Mocrobiol. 2007; 1-8.

44. Qin J, Zhao B, Wang X, Wang X, Wang L, Yu B, et al. Non-Sterilized Fermentative Production of Polymer-Grade L-Lactic Acid by a Newly Isolated Thermophilic Strain Bacillus sp. 2-6. Plos One 2009; 4(2):e4359.

45. Batdorj B, Trinetta V, Dalgalarrondo M, Prévost H, Dousset X, Ivanova I, et al. Isolation, taxonomic identification and hydrogen peroxide production by Lactobacillus delbrueckii subsp. lactis T31, isolated from Mongolian yoghurt: inhibitory activity on food-borne pathogens. J. Appl. Microbiol. 2007; 103:584-593. 